\title{
ARTICLE
}

\section{Five tips for good office spirometry}

D M Maree, National Diploma in Clinical Technology (Pulmonology and Critical Care)

Lung Unit, Division of Pulmonology, Department of Medicine, Faculty of Medicine and Health Sciences, Stellenbosch University and Tygerberg Academic Hospital, Cape Town, South Africa

Corresponding author: D M Maree (david.maree@westerncape.gov.za)

1. Spirometry is critical for the correct diagnosis of chronic obstructive pulmonary disease (COPD) and is part of the severity classification. It ultimately guides treatment choices. When performing spirometry on a COPD patient, one expects a flow volume loop to have some degree of obstruction. To obtain and confirm this result, there are a few important steps that have to be followed and rules that have to be adhered to. ${ }^{[1,2]}$

2. The spirometer has to be adjusted for atmospheric variables, i.e. temperature, humidity and barometric pressure. These variables are necessary to adjust the correction factor of the spirometer for volume measurement at BTPS (body temperature and pressure, saturated), as the inhaled air is at ambient conditions but the exhaled air is at body environment conditions. The spirometer also has to be calibrated daily, or prior to patients being tested, by means of a $3 \mathrm{~L}$ calibrating syringe. This calibration and variable adjustment ensure correct/accurate measurement.

3. The patient's demographics must be entered into the spirometer: age, height (in socks/barefoot), gender, race and weight, as these are used to calculate the predicted values for each individual. Without the correct predicted values, the resultant spirometric grading and the GOLD COPD classification ${ }^{[3]}$ will be incorrect, which will result in the patient being managed inappropriately.

4. When performing the test on a patient, each attempt (minimum - 3; maximum - 8) has to be acceptable in the following ways:

- At the start, the exhalation must be forced out as hard and fast as possible, without any hesitation.

- The minimum exhalation time is 6 seconds, although the more severe the COPD (a disease of airflow limitation) the longer the patient needs to exhale their full vital capacity; this could take up to 15 seconds. One should be extremely cautious at this point, as the patient could have a syncopal attack and collapse. Therefore, it is advisable to always perform the spirometry with the patient seated on a chair with armrests.

- There must be no artefacts such as coughing during the expiratory phase of the test, especially during the first 2 seconds, as this will affect the forced expiratory volume at 1 second $\left(\mathrm{FEV}_{1}\right)$. As coughing (and excessive phlegm production) is one of the symptoms of COPD, there is a strong possibility that the patient may have coughing bouts during the exhalation period. Any coughing after the first second has no effect on the grading of the spirometry and only affects the flow parameters.

- If the patient inserts his/her tongue into the mouthpiece, it causes an obstruction, resulting in abnormal flows and an erroneous $\mathrm{FEV}_{1}$, with a resultant incorrect COPD grading.

- If the patient does not maintain an adequate seal around the mouthpiece, flow of air passes around outside of the measuring device, leading to volume loss and hence the incorrect forced vital capacity (FVC). This will result in a flow volume loop that could be interpreted as a restrictive lung disease that is not in keeping with COPD, and the patient being incorrectly graded.

5. With the 3 acceptable attempts having been obtained, reproducibility is controlled by ensuring that the largest FVC and FEV are within $150 \mathrm{~mL}$ of each other. In severely impaired COPD patients, there is a possibility of reduced vital capacity of $<1 \mathrm{~L}$. If this occurs, the two largest FVCs and $\mathrm{FEV}_{1}$ s have to be within $100 \mathrm{~mL}$ of each other.

It is most important for spirometry and with the classification of the COPD patient that the procedure must also be performed after an adequate dose of a bronchodilator, i.e. post bronchodilator $\mathrm{FEV}_{1}$. This is required to standardise the assessment across patients and visits. Administering $400 \mu \mathrm{g}$ of salbutamol by means of a spacer is most effective, with a waiting period of at least 15 minutes before attempting the spirometry.

\section{References}

1. Koeglenberg CFN, Swart F, Irusen EM. Guideline for office spirometry in adults, 2012. S Afr Med J 2013;103(1):52-61. [http://dx.doi.org/10.7196/samj.6197]

Miller MR, Hankinson J, Brusasco V, et al. Standardisation of spirometry. Eur Respir J 2005;26:319338. [http://dx.doi.org/10.1183/09031936.05.00034805]

3. Global Initiative for Chronic Obstructive Lung Disease (GOLD). Global Strategy for Diagnosis Management and Prevention of COPD. 2015. http://www.goldcopd.org (accessed 21 July 2015).

S Afr Med J 2015;105(9):791. DOI:10.7196/SAMJnew.8428 